\title{
Direção ou Coordenação? Repensando a representatividade institucional da Enfermagem
}

\author{
Direction or Coordination? Rethinking the institutional representativeness of Nursing \\ ¿Dirección o Coordinación? Repensando la representatividad institucional de la Enfermería
}

\section{Maria de Lourdes de Almeida', Maria Luíza Hexsel Segui", Elizabeth Bernardino'"', Marinelli Joaquim Méier"II, Aida Maris Peres"I}

\author{
'Universidade do Oeste do Paraná. Curso de Enfermagem. Mestranda do Programa de Pós-Graduação em Enfermagem. \\ Universidade Federal do Paraná. Curitiba-PR, Brasil. \\ "Hospital de Clínicas. Universidade Federal do Paraná. Mestranda do Programa de Pós-Graduação em Enfermagem. \\ Universidade Federal do Paraná. Curitiba-PR, Brasil. \\ I' Universidade Federal do Paraná. Programa de Pós-Graduação de Enfermagem. Curitiba-PR, Brasil.
}

Submissão: 21/03/2010 Aprovação: 18/07/2011

\section{RESUMO}

Trata-se de pesquisa descritiva, de abordagem qualitativa, do tipo documental, que teve como local o Hospital das Clínicas da Universidade Federal do Paraná (HC-UFPR), na cidade de Curitiba-PR. O objetivo foi analisar comparativamente a descrição de dois cargos que representam o Serviço de Enfermagem, adotados em modelos gerenciais distintos. A análise de conteúdo dos documentos referentes aos cargos nos respectivos modelos de gerência evidenciou diferenças significativas. Os resultados apontaram várias alterações na descrição dos cargos e, dentre elas, do termo Direção de Enfermagem para Coordenação de Enfermagem, com a supressão da autonomia e poder de decisão sobre assuntos inerentes à profissão. Conhecimento e competência no exercício profissional são condições para que o enfermeiro mantenha sua autonomia e representatividade na estrutura organizacional.

Descritores: Enfermagem; Prática profissional; Organização e Administração; Estrutura Organizacional.

\section{ABSTRACT}

This is a documentary-type descriptive research, using a qualitative approach and, which was carried out at the Hospital of the Federal University of Paraná (HC-UFPR), in the city of Curitiba-PR. It aimed to comparatively analyze the description of two job positions that represent the nursing service, which were adopted in different managerial models. The content analysis of the documents related to the job positions in the respective management models showed significant differences. The results pointed out several changes in the description of the job positions, i.e. the term Nursing Direction was changed into Nursing Coordination, involving the suppression of autonomy and decision-making power over matters intrinsic to this profession. Knowledge and competence in the professional practice are required by the nurse practitioners in order to keep their representativeness in the organizational structure.

Key words: Nursing; Professional Practice; Organization and Management; Organizational Structure.

\section{RESUMEN}

Se trata de una investigación descriptiva, de abordaje cualitativa, de tipo documental, que tuvo como local el Hospital de Clínicas de la Universidad Federal de Paraná (HC-UFPR), en la ciudad de Curitiba-PR. El objetivo fue analizar comparativamente la descripción de dos cargos que representan al servicio de Enfermería, adoptados en modelos gerenciales diferentes. El análisis del contenido de los documentos referentes a los cargos en los respectivos modelos de gerencia puso en evidencia diferencias significativas. Los resultados apuntaron varias alteraciones en la descripción de los cargos, entre ellas el término Dirección de Enfermería por Coordinación de Enfermería, con la supresión de la autonomía y poder de decisión sobre asuntos inherentes a la profesión. Conocimiento y competencia en el ejercicio profesional son condiciones para que el enfermero mantenga su autonomía y representatividad en la estructura organizacional.

Palabras clave: Enfermería; Práctica Profesional; Organización y Administración; Estructura Organizacional. 


\section{INTRODUÇÃO}

Vive-se um período de significativas transformações gerenciais, instabilidade social e econômica. Nesta situação, as instituições de saúde, principalmente os hospitais, trabalham na reestruturação de seus modelos de gerenciamento, que incidem também nos Serviços de Enfermagem.

As alterações institucionais afetam a prática gerencial do enfermeiro, que, por consequência, influencia a prática do cuidado. As funções administrativas de planejamento, organização, direção e avaliação exercidas por este profissional ocupam lugar de destaque no trabalho da equipe de saúde. É neste cenário que o enfermeiro deve apoiar o seu fazer, utilizando-se de novas tecnologias gerenciais, a fim de tornar-se um agente de mudança da realidade dos Serviços de Enfermagem das instituições de saúde.

Historicamente, o gerenciamento de enfermagem vem sofrendo alterações na tentativa de utilizar meios mais eficazes que adaptem os modelos administrativos ao seu processo de trabalho, focando sempre o cuidado ao paciente ${ }^{(1)}$.

Com isso, ressalta-se a importância de o profissional ter conhecimento de como o seu trabalho é produzido e consumido, assim como sua relevância no contexto social, tendo em vista que o trabalho da enfermagem não produz bens a serem estocados e seus serviços são consumidos no momento da assistência ${ }^{(2)}$. Os cuidados realizados são o produto do seu serviço e, quando o cliente busca tratamento e cuidados, requer vários insumos, como pessoal capacitado, material e informações, no processo de produção caracterizado pela prestação de serviços de enfermagem.

Compete ao enfermeiro, conforme descreve a Lei do Exercício Profissional de Enfermagem, as funções de planejamento, coordenação, execução e avaliação da assistência de Enfermagem, sendo o gerenciamento uma face do seu processo de trabalho( ${ }^{(3)}$. Assim, ele é o responsável pelo trabalho da equipe de Enfermagem e também pelo gerenciamento do cuidado, sendo este trabalho desenvolvido em diferentes cargos e funções que o enfermeiro ocupa nas instituições prestadoras de cuidados a saúde.

A Enfermagem é a única profissão da área da saúde que, em todos os espaços, permanece 24 horas com o paciente e é ela, no papel do enfermeiro, quem coordena o cuidado prestado pelos outros profissionais aos pacientes ${ }^{(4)}$. Seu papel "quase silencioso" no cotidiano garante, pela sua competência profissional no processo gerencial, os insumos indispensáveis ao cuidado, articula e encaminha os procedimentos necessários à realização de exames complementares, supervisiona as condições de hotelaria, conversa com a família, administra a circulação do paciente entre as diferentes áreas do hospital e responde por um número significativo de atividades que resultam no cuidado ${ }^{(5)}$.

Recentemente, alguns hospitais alteraram seus modelos gerenciais na busca de se adequar ao modelo assistencial proposto pelo Sistema Único de Saúde (SUS), e na procura da sua integração a este sistema, foram necessárias várias modificações tanto conceituais como operacionais na sua forma de gerenciamento(6). Estes hospitais mudaram suas lógicas e tentam encontrar modelos ancorados no trabalho multidisciplinar, com a intenção de oferecer uma assistência de melhor qualidade aos seus usuários, na perspectiva da consolidação dos princípios do $S U S^{(4)}$.

Essa reestruturação dos modelos de gestão decorre da crescente complexidade dos processos de produção de cuidados à saúde e representa um desafio na forma como esses profissionais se organizam para prestar a assistência ${ }^{(7)}$.

A organização do trabalho na lógica de que todos os componentes da equipe de saúde têm a mesma importância e, portanto, todos são aptos a exercer sua liderança. Esta mudança assinala certa tendência em relativizar as especificidades das profissões, em geral, e da Enfermagem em particular, com ênfase na natureza complementar do trabalho em equipe. Essa nova forma de "fazer" o trabalho sugere mudanças na forma de "pensar" o trabalho: a interdisciplinaridade propõe a interação de profissionais de diferentes disciplinas, conservando as especificidades de cada uma e de seus membros, para a construção de um trabalho coletivo ${ }^{(8)}$.

Para cada modelo assistencial, há um padrão gerencial e uma operacionalização organizacional, traduzidos em cargos e funções. A dimensão mais visível das relações de poder existentes em uma organização é aquela expressa nos arranjos hierarquizados de autoridade, do tipo legal-formal, que se mostra nos organogramas formais, nos regulamentos e nos rituais do cargo(4).

Em 2002, ocorreu uma mudança no Hospital de Clínicas da Universidade Federal do Paraná (HC-UFPR), quando o grupo diretivo mudou seu modelo gerencial, construído em uma lógica de profissões, para um modelo na lógica de equipe ${ }^{(6)}$. Ao operacionalizar esta proposta, houve a substituição da Direção de Enfermagem por uma Coordenação de Enfermagem. Esta alteração aconteceu ancorada, principalmente, nos conceitos e nos pressupostos embutidos nos modelos gerenciais aos quais eles estavam atrelados, seguindo a lógica de "unidades de produção ou unidades funcionais".

Uma pesquisa nesta instituição mostrou que a mudança de modelo e a substituição da Direção por uma Coordenação foi traumática para a maioria das enfermeiras, porque representou a perda de poder e capital simbólicos, a falta de reconhecimento e de identidade profissional, que importaram em significativas perdas para a categoria( ${ }^{(6)}$.

Diante do exposto, o presente estudo justifica-se por poder instrumentalizar a Equipe de Enfermagem na discussão de medidas que levem ao reconhecimento deste novo contexto institucional, contribuindo com discussões que possam ser relevantes para a (re)construção da gerência de enfermagem. Considerando que a categoria de Enfermagem é representada na instituição por um cargo, partiu-se do pressuposto de que sua descrição acompanha o modelo gerencial e o espaço ocupado por ela. Assim, o objetivo deste estudo é analisar comparativamente a descrição dos dois cargos do Serviço de Enfermagem adotados em modelos gerenciais distintos. Esta análise será feita pela comparação das atribuições da Direção de Enfermagem definidas na gestão anterior e da Coordenação de Enfermagem no modelo atual.

\section{MÉTODO}

Trata-se de uma pesquisa descritiva, de abordagem qualitativa, do tipo documental quanto à coleta de dados. A pesquisa 
descritiva tem como objetivo a descrição das características de determinada população ou fenômeno ou o estabelecimento de relações entre as variáveis e a pesquisa documental utiliza materiais que ainda não receberam tratamento analítico, ou podem ser reelaborados de acordo com o objeto de pesquisa ${ }^{(9)}$.

O local da pesquisa foi o HC-UFPR, localizado na cidade de Curitiba, capital do Estado do Paraná. Para a análise documental, utilizou-se a descrição das competências do cargo de Diretor de Enfermagem do modelo gerencial anterior a 2002 e a descrição das atribuições do cargo de Coordenador de Enfermagem do modelo gerencial posterior a 2002 ${ }^{(10-11)}$. A comparação dos documentos relacionados à mudança no modelo gerencial da instituição foi operacionalizada pela análise de conteúdo ${ }^{(12)}$.

O projeto da pesquisa foi submetido ao Comitê de Ética em Pesquisa do HC-UFPR, em atendimento à Resolução no 196/1996, do Ministério de Saúde, que versa sobre Diretrizes e Normas Regulamentadoras de Pesquisa envolvendo Seres Humanos. O projeto foi aprovado pela Parecer $\mathrm{n}^{\circ}$ 0194.0.208.000-09. Foi também solicitada à Direção do HC/ UFPR a disponibilização de documentos oficiais para a coleta de dados da pesquisa.

\section{RESULTADOS E DISCUSSÃO}

Após a leitura dos documentos relacionados à mudança no modelo gerencial da instituição, foi possível apreender as atribuições dos referidos cargos, bem como sua adesão à linha gerencial proposta pelo hospital, que reflete o modelo assistencial vigente no país. Assim, antes da apresentação de uma súmula de cada cargo, é descrito quando o cargo foi instituído e sua posição no organograma institucional, sempre buscando localizar a representação da Enfermagem nos dois cenários. Após a descrição dos cargos, é apresentada uma análise comparativa dos principais aspectos encontrados.

\section{Descrição do cargo de Direção de Enfermagem}

O cargo de Direção de Enfermagem do hospital foi criado em 1981 e, no organograma geral, estava subordinado à Direção Geral, com o mesmo nível hierárquico das outras direções e participação do plano orçamentário do hospital. O regulamento da instituição era seguido, e seus projetos deviam estar em consonância com os da Direção Geral; anualmente, o planejamento e o relatório das atividades eram encaminhados ao Conselho de Administração da Instituição.

Dentre as competências da Direção de Enfermagem ${ }^{(10)}$, encontravam-se: 1) planejamento das estratégias político-filosóficas relacionadas ao ensino; 2) administração, assistência e pesquisa em enfermagem; 3) gerenciamento de recursos humanos: dimensionamento, avaliação do desempenho do profissional, movimentação de pessoal, Educação Permanente em Enfermagem, com os programas de educação em serviço, motivação e satisfação da equipe no trabalho; 4) desenvolvimento e implantação da Sistematização da Assistência de Enfermagem; 5) auditoria da qualidade e avaliação do Serviço de Enfermagem; 6) participação no planejamento de aquisição de material permanente e de consumo para o serviço de enfermagem; 7) articulação da integração entre a assistência e a docência, intra e extra-instituição; 8) possibilitar a participação da equipe de Enfermagem na indicação dos coordenadores de área e chefias de serviço; e 9) colaboração, por meio de estudos e projetos, com os demais serviços do hospital visando ao alcance dos objetivos do plano diretor.

A Direção de Enfermagem estava na mesma linha hierárquica das outras direções e ocupava assento e direito a voto nos fóruns de discussão e deliberação, tanto no Conselho Administrativo como no Conselho Diretivo do hospital. Também teve função executiva e normativa, representatividade junto à direção geral, órgãos colegiados e órgãos de classe. No documento consultado fica claro que a Direção de Enfermagem seguia o modelo político e gerencial da instituição.

\section{Descrição do cargo de Coordenação de Enfermagem}

A Coordenação de Enfermagem foi criada em 03 de fevereiro de 2003 pela Resolução n003/2003 do Conselho de Administração do Hospital de Clínicas da Universidade Federal do Paraná(11). É normativa, não possui linha de mando nesse novo contexto e, como Coordenação, não está representada no organograma (modelo horizontal). A Coordenação de Enfermagem esteve, desde sua instituição, subordinada a várias direções, como a Direção de Ensino e Pesquisa, a Direção de Corpo Clínico e, atualmente, a Direção de Assistência.

Conforme a Resolução n003/2003 da Direção de Corpo Clínico, a Coordenação de Enfermagem tem como atribuições ${ }^{(11)}:$ 1) função de assessoramento dos gerentes das Unidades Funcionais na organização, planejamento e avaliação dos processos de enfermagem, assim como sua implantação; 2) realizar os programas e atividades de treinamento, aperfeiçoamento e desenvolvimento de profissionais de enfermagem, em conjunto com a Unidade de Administração de Pessoas; 3) manter avaliação técnica contínua dos processos de enfermagem, rever continuamente os padrões de assistência de enfermagem do Hospital e propor seu aperfeiçoamento; 4) prestar serviços de consultoria e emitir parecer técnico de enfermagem quando necessário; 5) conhecer e avaliar os programas de treinamento na área de enfermagem de pós-graduação, de graduação e de nível médio das instituições conveniadas, adequando-os às demandas da instituição; e 6) promover estudos e pesquisas no campo da Enfermagem, visando à melhoria da assistência e do nível de conhecimento técnico/ científico da equipe.

A Coordenação de Enfermagem participa, com direito a voto, do Conselho Diretivo do hospital. Este Conselho é formado pelos diretores, gerentes e coordenadores. No entanto, não participa do Conselho de Administração do HC/UFPR, órgão decisório superior no organograma institucional.

\section{Análise comparativa entre Direção e Coordenação}

Ao comparar os dois cargos que representam o Serviço de Enfermagem, nos dois modelos gerenciais distintos, percebem-se diversas mudanças na transposição de um para o outro. Pode-se citar primeiramente que a mudança de Direção de Enfermagem para Coordenação de Enfermagem tem significados diferentes, tanto do ponto de vista semântico, como da representatividade. 


\begin{tabular}{|l|l|l|}
\hline \multicolumn{1}{|c|}{ ITEM DE ANÁLISE } & \multicolumn{1}{|c|}{$\begin{array}{c}\text { MODELO GERENCIAL } \\
\text { ANTERIOR }\end{array}$} & $\begin{array}{c}\text { MODELO GERENCIAL } \\
\text { ATUAL }\end{array}$ \\
\hline Denominação & Direção de Enfermagem & Coordenação de Enfermagem \\
\hline Organograma & Ligada à Direção Geral & Não consta \\
\hline Representatividade & $\begin{array}{l}\text { Assento e voto no Conselho Diretivo } \\
\text { e Conselho Administrativo }\end{array}$ & Assento e voto no Conselho Diretivo \\
\hline Termo adotado & Competência & Atribuição (conceito voltado à tarefa) \\
\hline Função principal & Executiva & Normativa \\
\hline Forma de gerenciamento & Centralizada & Descentralizada \\
\hline $\begin{array}{l}\text { Relação com outras } \\
\text { categorias profissionais }\end{array}$ & Centrada na Enfermagem & Trabalho em equipe multiprofissional \\
\hline Planejamento & Recursos humanos & Não consta \\
\hline
\end{tabular}

Fonte: Hospital de Clínicas da Universidade Federal do Paraná, 1999; Hospital de Clínicas da Universidade Federal do Paraná, 2003.

Quadro1 - Comparação entre os cargos de Direção e de Coordenação de Enfermagem

A Direção de Enfermagem ocupou uma posição definida no organograma, estando ligada à Diretoria Geral do Hospital. Entretanto, a Coordenação de Enfermagem não consta no organograma, aspecto que afeta sua representatividade. Em estudo realizado em 2006, relatou-se que, quando da extinção da Direção de Enfermagem, a autonomia do enfermeiro foi afetada, já que esta autonomia servia de referência para as questões executivo-deliberativas na resolução dos problemas deste grupo de profissionais ${ }^{(13)}$.

$\mathrm{Na}$ análise dos documentos que descrevem os cargos, notam-se várias distinções. Pode-se pontuar inicialmente a diferença entre a palavra direção e coordenação para a denominação do Serviço de Enfermagem. O termo direção indica o ato ou efeito de dirigir (-se); comando, liderança, conjunto de esforços com o objetivo de formação, gestão de negócios públicos ou privados; administração, gerenciamento, indivíduo ou grupos que exercem a função administrativa (em uma instituição, empresa, órgão público etc.); diretor, diretoria, cúpula (14). Assim, a função de direção está relacionada diretamente com o planejamento organizacional, o alcance dos objetivos traçados, a condução e coordenação de recursos humanos e se refere às relações interpessoais em todos os níveis da organização e de seus respectivos subordinados.

Para o termo coordenação, tem-se como alguns dos significados: ato ou efeito de coordenar(-se); ato de conjugar, concatenar um conjunto de elementos, de atividades etc.; estado daquilo que está coordenado, gerência de determinado projeto, setor; entre outros ${ }^{(14)}$.

Na descrição das funções dos cargos de Direção e Coordenação, encontram-se "Competência da Diretoria de Enfermagem" e "Atribuições da Coordenação de Enfermagem". Aqui já fica evidente a mudança no conceito entre um modelo e outro, no que se refere ao significado das palavras. Esta denominação reflete a forma da estruturação do Serviço de Enfermagem e impacta a percepção dos profissionais sobre este serviço. Competência pode ser definida como um conjunto de conhecimentos, habilidades e atitudes que permitem ao enfermeiro identificar e acessar informações determinantes para a fundamentação de suas atitudes ${ }^{(15)}$.

No caso da Direção, estas competências são as teorias administrativas, as ferramentas específicas da gerência, o processo de trabalho, a ética no gerenciamento, conhecimentos sobre cultura e poder organizacional, negociação, trabalho em equipe, qualidade de vida no trabalho, saúde do trabalhador, leis trabalhistas, gerenciamento de pessoas, dimensionamento de pessoal, gerenciamento de recursos materiais, custos, recursos físicos, sistemas de informação e processo decisório ${ }^{(15)}$.

Lembrando que as competências agregam valor econômico para a organização, devem também agregar valor social ao indivíduo, ou seja, as pessoas, ao desenvolverem competências essenciais para o sucesso da organização, estão também investindo em si mesmas, não só como cidadãos organizacionais, mas como cidadãos do próprio país e do mundo ${ }^{(16)}$. Já o conceito de atribuição, destinado à Coordenação de Enfermagem, está relacionado com o "fazer" tarefeiro, pois leva ao ato de atribuir, de encargo.

A Direção teve a função executiva e a Coordenação é somente normativa. As questões político-administrativas eram centralizadas na Direção e a Coordenação não tem esta função e, como está descrito no documento, outros setores se encarregam deste trabalho. A Coordenação está inserida na lógica do trabalho multidisciplinar, privilegiando o trabalho 
em equipe. A Direção estava mais voltada à Enfermagem, cujo fluxo das relações hierárquicas era vertical, característica do organograma e da hierarquização tradicional.

O gerenciamento do hospital e da Enfermagem seguia o paradigma positivista, fundamentado na Teoria Geral da Administração, com a valorização da hierarquia, com evidência na estrutura formal da organização e na centralização das decisões, impessoalidade nas relações, morosidade nas decisões e fluxo de comunicação verticalizado ${ }^{(13)}$.

Apesar de prescritiva e seguir um modelo tradicional de gerenciamento, a Direção teve muitas funções participativas. O documento com as atribuições da Coordenação não traz possibilidades de autonomia participativa para este órgão, quando determina que deve visar à normatização, controle ético-profissional e de finalidade de atendimento do corpo de Enfermagem.

Nas atribuições da Coordenação não aparecem o dimensionamento de pessoal, a motivação e satisfação no trabalho, importantes instrumentos de gestão para o enfermeiro apoiar sua prática gerencial. O dimensionamento de pessoal em enfermagem é foco da atenção dos enfermeiros ,já que a previsão adequada da equipe, tanto no aspecto quantitativo como no qualitativo, objetiva a prestação de cuidados com segurança e qualidade à clientela assistida ${ }^{(17)}$.

Outra diferença observada é no quantitativo dos itens que descrevem os cargos. Houve uma redução na descrição do cargo de Coordenação de Enfermagem, pois suas competências são descritas em vinte e cinco itens, e suas atribuições são especificadas em apenas onze. Esse resultado leva-nos a questionar se, ao mesmo tempo em que o Serviço de Enfermagem muda de denominação, diminui sua representatividade e a necessidade de detalhar suas atividades.

A avaliação de desempenho da equipe e da assistência de enfermagem é uma atividade privativa do enfermeiro, segundo sua lei do exercício profissional. No caso da Coordenação de Enfermagem o enfermeiro perde esta autonomia na medida em que outras categorias de profissionais são responsáveis por esta atividade.

A Enfermagem ocupa importante espaço no grupo de recursos humanos das organizações hospitalares, devido ao seu significativo número de profissionais. Sendo assim, a avaliação de desempenho focada no processo de trabalho é utilizada para fazer uma abordagem adequada da equipe no que se refere à sua produção e realização profissional ${ }^{(18)}$.

\section{CONSIDERAÇÕES FINAIS}

Percebe-se que, no cargo de Direção de Enfermagem, estava implícito um poder formal e instituído, no qual o dirigente era valorizado pelos profissionais ,que lhe concediam confiança mediante as evidências de sua autoridade legal. A Direção de Enfermagem foi referência para a equipe de enfermagem nas questões executivo-deliberativas e também na resolução de problemas do Serviço de Enfermagem. Teve, ain$\mathrm{da}$, a finalidade de estabelecer diretrizes norteadoras para a atuação dos profissionais, direcionando sua prática com vistas à qualidade da assistência.

Com o novo modelo, surgiu a proposta de descentralização das decisões no HC/UFPR, sustentada por uma linha gerencial compatível com os princípios do sistema de saúde vigente no país. Mas, para a Enfermagem, esse advento representou diferentes encaminhamentos, entre os quais: desarticulação entre os profissionais da área, falta de padronização dos processos de trabalho e de procedimentos de enfermagem da instituição.

Hoje, algumas funções específicas do enfermeiro, respaldadas legalmente pela Lei do Exercício Profissional de Enfermagem, estão a cargo de outros profissionais, como o dimensionamento, movimentação de recursos humanos, atividades de treinamento, aperfeiçoamento e desenvolvimento dos profissionais de Enfermagem.

É necessário que os profissionais de enfermagem adquiram conhecimentos e desenvolvam competências para criar e manter as condições propícias para a realização do cuidado. O trabalho em equipe e a valorização do trabalho de outros profissionais devem considerar o contexto atual de desapego ao poder. Mas tal proposta não pode ser concretizada em detrimento do exercício dos direitos e capacidades dos enfermeiros, que os tornam aptos a agir com autonomia.

A menor representatividade na estrutura organizacional e a descontinuidade administrativa podem provocar o enfraquecimento da enfermagem e possibilitar a interferência externa, sem compreensão do processo de trabalho da categoria, especialmente no que diz respeito à distribuição de recursos humanos e definição de prioridades para o cuidado.

\section{REFERÊNCIAS}

1. Jorge MSB, Freitas, CHA, NMFB, Queiroz MVO. Gerenciamento em Enfermagem: um olhar crítico sobre o conhecimento produzido em periódicos brasileiros (20002004). Rev Bras Enferm 2007 fev; 60(1): 81-86.

2. Felli VEA, Peduzzi M. O trabalho gerencial do enfermeiro. In: Kurcgant P, coordenadora. Gerenciamento em enfermagem. Rio de Janeiro: Guanabara Koogan; 2005.

3. Presidência da República (BR). Decreto $n^{\circ} 94.406$ de 08 de Junho de 1987. Regulamenta a Lei $n^{\circ} 7.498$ de 25 de junho de 1986, que dispõe sobre o exercício da enfermagem, e dá outras providências. Diário Oficial da União 1987.

4. Cecílio LCO, Mendes TC. Propostas alternativas de gestão hospitalar e o protagonismo dos trabalhadores: por que as coisas nem sempre acontecem como os dirigentes desejam? Saúde soc 2004 maio/ago; 13(2):39-55.

5. Cecilio LCO, Merhy EE. Integralidade do cuidado como eixo da gestão hospitalar. Campinas; 2003. [citado 13 maio 2009]. Disponível em: www.hc.ufmg.br/gids/Integralidade.doc 
6. Bernardino E, Felli VEA. Saberes e poderes necessários à reconstrução da enfermagem frente a mudanças gerenciais num hospital de ensino. [citado 22 maio 2009]. Rev Latino-am Enfermagem 2008 nov-dez; 16(6). Disponível em: www.eerp.usp.br/rlae

7. Magalhães AMM, Riboldi CO, Dall 'Agnoll CM. Planejamento de recursos humanos de enfermagem: desafio para as liderenças. Rev Bras Enferm 2009 jul-ago; 62(4):608-12.

8. Bernardes A, Évora YDM, Nakao JRS. Gestão colegiada na visão dos técnicos e auxiliares de enfermagem em um hospital público brasileiro. Ciencia y Enfermeria 2008; 14(2): 65-74.

9. Gil AC. Como elaborar projetos de pesquisa. 4.ed. São Paulo: Atlas; 2008.

10. Hospital de Clínicas da Universidade Federal do Paraná. Direção de Enfermagem. Competências da Direção de Enfermagem. Curitiba: HC/UFPR, 1999. Manuscrito.

11. . Resolução no 003/2003-COAD/HC/UFPR. Aprova as Atribuições da Coordenação de Enfermagem. Curitiba: HC/UFPR, 2003.

12. Minayo MCS, Deslandes SF, Gomes R. Pesquisa social: teoria, método e criatividade. 27.ed. Petrópolis/RJ: Vozes; 2008.

13. Bernardino E, Felli VEA. A organização da Enfermagem do HC-UFPR: refletindo sobre seus determinantes. Rev Bras Enferm 2006 abr; 59(2):148-53.

14. Instituto Antonio Houaiss. Dicionário eletrônico Houaiss da língua portuguesa, versão 2.0. São Paulo: Objetiva; 2006.

15. Peres AM, Ciampone MHT. Gerência e competências gerais do enfermeiro. Texto \& Contexto Enferm 2006 jul/set; 15(3):492-9.

16. Fleury MTL, Fleury A. Construindo o Conceito de Competência. RAC 2001; Edição Especial: 183-96.

17. Wolff LDG, Mazur CS, Wiezbicki C, Barros CB, Quadros VAS. Dimensionamento de pessoal de enfermagem na unidade semi-intensiva de um hospital universitário de Curitiba. Cogitare Enferm. 2007 abr/jun; 12(2):171-82.

18. Brahm MMT, Magalhaes AMM. Opinião da equipe de enfermagem sobre o processo de avaliação de desempenho. [citado 13 maio 2009]. Acta paul. enferm. [online] 2007;20(4): 415-21. 